\title{
Immediate Effect of Lesion of the Ventromedial Hypothalamic Area upon Glucose-Induced Insulin Secretion in Anaesthetized Rats
}

\author{
F. Rohner, A.-C. Dufour, C. Karakash, Y. Le Marchand, K. B. Ruf ${ }^{1}$, and B. Jeanrenaud \\ Laboratoires de Recherches Médicales, Départements de Médecine et de Physiologie ${ }^{1}$, Université de Genève, Genève, Switzerland
}

\begin{abstract}
Summary. Insulin secretion, measured in vivo following an intravenous load of glucose to anaesthetized rats, was markedly increased ten minutes after bilateral electrolytic lesions of the ventromedial hypothalamic (VMH) area when compared to both sham-operated and unoperated controls. The successful lesioning of the VMH area was assessed by the subsequent occurrence of hyperphagia, as estimated by the increase in body weight. It is concluded that the ventromedial hypothalamic area exerts an inhibitory influence upon the secretory activity of the B-cells. Furthermore, the rapid disappearance of such inhibitory influence following lesions of the VMH suggests that this area of the brain may be of importance in the minute to minute regulation of insulin secretion. The precise anatomical location of the hypothalamic "nucleus" (or "nuclei") involved, as well as the neural or humoral nature of its inhibitory effect upon the endocrine pancreas remain to be elucidated.
\end{abstract}

Key words: Ventro-medial hypothalamus area (VMH), insulin secretion, rat, VMH insulin relationship

It is well established that the destruction of the ventromedial hypothalamic (VMH) area by local electrolysis produces chronic hyperphagia and hyperinsulinaemia in rats, the latter being usually assessed days after the surgical procedure [1-2]. However, hyperinsulinaemia does not appear to be a simple consequence of hyperphagia, since it has been shown to exist in VMH-lesioned animals that are not hyperphagic [2-5] or in which hyperphagia has been prevented by appropriate food restriction [6,
7]. Hyperinsulinaemia thus represents a genuine change that follows a lesion of the ventromedial hypothalamic area. Hyperinsulinaemia is also likely to be of importance in experimental obesity since it has been reported that the weight gain of rats with VMH lesions could be predicted from the increase of basal insulin levels that occurred after the lesion [8]. The likelihood that the obesity of rats with VMH lesions is indeed related to the activity of the $B$-cells was further substantiated by the observation that such rats did not become obese when made insulin-deficient [9]. It has been demonstrated that insulin secretion by the B-cells is under the control of the autonomic nervous system, both sympathetic and parasympathetic nerves modulating the output of the hormone $[10,11]$. However, the precise relationship that may exist between the hypothalamus and the autonomic nervous system is not known. It is interesting, in this respect, to note that the obesity which follows lesions of the VMH can be largely prevented by sectioning the vagus nerves [12]. Furthermore, it has been suggested that the VMH area could exert an inhibitory influence on the ventrolateral hypothalamic (VLH) area which would normally promote insulin secretion (for review, see [2]). It has also been claimed that a factor exists in the VLH area that stimulates insulin release by pancreatic islets $[13,14]$. A neural relationship between the hypothalamus and the secretory activity of the B-cells has also been suggested: insulin secretion by normally innervated, vascularly isolated, in situ rat pancreas was reported to increase following electrical stimulation of the hypothalamus [15].

In the present study, we have tested the hypothesis that if an inhibitory influence of the hypothalamus, be it neural or humoral, were to be of relevance in the acute regulation of peripheral insulin concentration, it should rapidly disappear fol- 


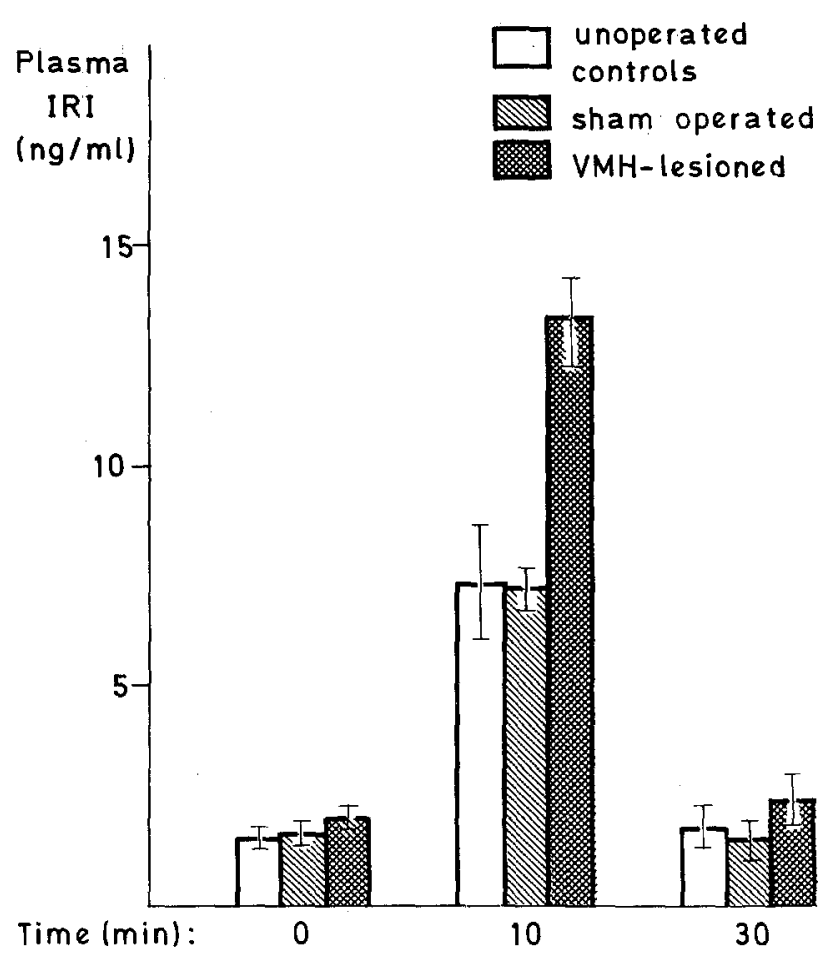

Fig. 1. Plasma insulin concentrations following intravenous glucose load in normal, sham-operated and acutely ventromedial hypothalamus (VMH)-lesioned rats. Unoperated, sham-operated or VMH-lesioned rats immediately following the surgical procedure, were given an IV glucose load $(0.6 \mathrm{~g} / \mathrm{kg}$ body wt). Each bar is the mean of 6 (unoperated controls); 7 (sham-operated); or 10 (VMH-lesioned) rats \pm SEM. Insulinaemia at $10 \mathrm{~min}$ : VMHlesioned rats differed from unoperated or sham-operated controls at $\mathrm{p}<0.01$

lowing destruction of the $\mathrm{VMH}$ area. We have therefore investigated the acute consequence of electrolysis on this secretion in vitro.

\section{Materials and Methods}

\section{Animals}

Ten week old female Wistar rats weighing $220 \mathrm{~g}$ and bred in these laboratories were used. They were placed in animal quarters with constant temperature $\left(23^{\circ} \mathrm{C}\right)$ and fixed $(12 \mathrm{~h})$ light cycle. The animals were fed ad libitum with UAR laboratory chow (Villemoisson, Epinay/Orge, France) up to the beginning of the experiment (9-11 a.m.).

\section{Experimental design}

Rats were anaesthetized by intraperitoneal injection of pentobarbital (50 mg per kilo body wt). A polyethylene catheter ( $p$ p 50, Portex, England) was then introduced into the external jugular vein and used for subsequent blood sampling. The animals were subdivided into three groups: untreated, shamoperated, and VMH-lesioned rats. Electrolytic lesions (David Kopf Instruments, Model 900) were placed stereotaxically by passing an anodal d.c. current $(0.8 \mathrm{~mA}, 40 \mathrm{~s})$ through steel electrodes $(0.4$ $\mathrm{mm}$ in external diameter, and insulated by varnish except for a $0.5 \mathrm{~mm}$ free tip). Stereotaxic coordinates used for destroying the ventromedial hypothalamic $(\mathrm{VMH})$ area were $\mathrm{A}+6.2 \mathrm{~mm}, \mathrm{~L}+$ $1.1 \mathrm{~mm}$ and $\mathrm{H}-3.7$ (16). Sham-operated rats were treated as the lesioned animals, except that stereotaxic coordinates were different, i. e., $\mathrm{A}+8.1$ $\mathrm{mm}, \mathrm{L}+1.1 \mathrm{~mm}, \mathrm{H}-1.9$. No current was passed through these electrodes. Immediately after operation, the first blood samples were taken from the jugular vein. This was followed by a rapid injection, into the jugular vein of all rats, of a glucose bolus $(0.6 \mathrm{~g} / \mathrm{kg}$ body weight $)$. This procedure was used since preliminary results had shown that basal insulinaemia was not modified by an acute lesion to the VMH. Blood samples $(300 \mu l)$ were again taken 10 and 30 min later, and centrifuged. Supernatants were used for measurement of plasma immunoreactive insulin (IRI) [17], using rat insulin as standard. The lower limit of detection of the assay was 0.125 $\mathrm{ng} / \mathrm{ml}$. Plasma glucose levels were determined by the glucose oxidase method [18]. After the experiment, animals were kept alive, and the adequacy of the lesions was assessed on the basis of weight gain over subsequent days. Only those rats with successful VMH lesions were taken into consideration.

\section{Results}

As shown in Figure 1, basal insulin concentration was not modified immediately after the lesion of the ventromedial area of the hypothalamus, remaining at values that were similar to those of untreated or sham-operated controls. In marked contrast, 10 minutes after an intravenous glucose bolus, the acutely lesioned animals had circulating insulin levels that were nearly twice those observed in controls. This difference was no longer present $30 \mathrm{~min}-$ utes later. Plasma glucose levels at 0,10 and $30 \mathrm{~min}$ following glucose administration were the same in untreated, sham-operated and lesioned rats, all plasma glucose concentrations in all groups having returned to basal values by $30 \mathrm{~min}$. One should emphasize in particular that the increased insulin secretion observed in the rats with VMH lesions when compared to controls was apparently in response to a similar glucose stimulation since, $10 \mathrm{~min}$ after glucose administration, the plasma glucose level was 
$372 \pm 60 \mathrm{mg} / 100 \mathrm{ml}(\mathrm{N}=7)$ for controls and $348 \pm$ $36 \mathrm{mg} / 100 \mathrm{ml}(\mathrm{N}=10)$ for rats with hypothalamic lesions. As illustrated in Figure 2, control or shamoperated rats lost weight the day following the experiment and did not gain any during the next five consecutive days. In contrast, animals with VMH lesions gained weight from the first post-operative day onwards. Among the lesioned rats, two failed to gain weight and actually lost 1 and 2 grams, respectively, the day after the experiment to remain thereafter within the body weight range of unoperated and sham-operated controls. As the lesion of the ventromedial hypothalamic area of these two rats was unsuccessful, data from these animals were not included in the results of Figures 1 and 2. It is of interest to note that in these two animals, the plasma insulin levels $10 \mathrm{~min}$ after the glucose load were 5.8 and $7.3 \mathrm{ng} / \mathrm{ml}$, respectively, i. e., well within the range observed in unoperated or sham-operated controls.

\section{Discussion}

These data show that a marked increase in insulin secretion following glucose administration in vivo can be detected within 10 minutes after electrolytic lesion of the ventromedial hypothalamic ( $\mathrm{VMH})$ area in anaesthetized rats prevented from having access to food. This is in contrast with a previous report indicating that the hyperinsulinaemia that followed VMH lesions was the result of an augmented food intake [19], an observation that can be attributed to a stimulatory influence of many different substrates and/or hormones. In the present study, the rapid occurrence in hypothalamic-lesioned rats of excessive insulin secretion (Fig. 1) was found to coincide with the subsequent development of hyperphagia as estimated by the increase in body weight that occurred within twenty-four hours and for the subsequent days. These observations strongly suggest that the hypothalamus and/or neighbouring structures have the ability to influence the amount of insulin secreted by the pancreas. This may indicate that the "satiety" centre is functionally related or anatomically close to the hypothalamic structures that, directly or indirectly, influence the secretion of insulin. It is also conceivable that these structures operate independently from those responsible for food intake since their electrolytic lesion resulted in acute alterations in plasma insulin levels clearly unrelated to change in food intake. Hyperinsulinaemia has been previously shown to occur in ventromedial hypothalamus-lesioned rats in the absence of any hyperphagia $[2,3,8]$. This study indicates, however, that the inhibitory influence of the hypothalamus

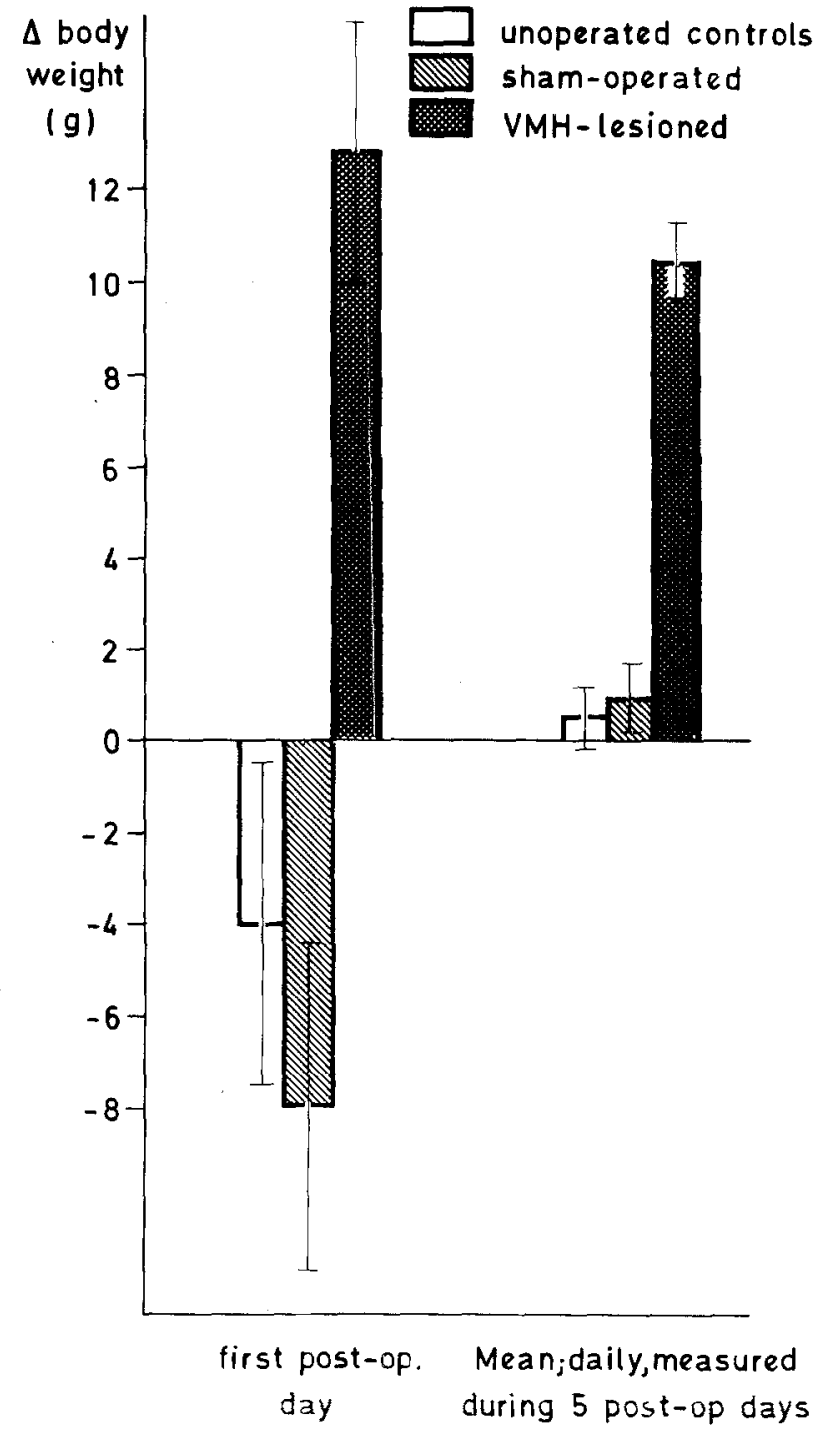

Fig. 2. Change in body weight of normal, sham-operated and ventromedial hypothalamus (VMH)-lesioned rats. Rats were treated as indicated in Figure 1. Increments in body weights were recorded the day after the experiments (left). Daily increments in body weights were recorded during 5 subsequent post-operative days, and averaged (right). Each bar is the mean of 6 (unoperated controls); 7 (sham-operated); or 10 (VMH-lesioned) rats \pm SEM

upon insulin secretion disappears immediately after the lesion. We have demonstrated elsewhere [7], using a similar in vivo test, the existence of abnormally high insulin secretion in non-hyperphagic rats used ten days after the lesion of the ventromedial hypothalamus. The increase in insulin levels observed in these rats ten minutes after glucose load was of the same order of magnitude as that recorded in the present experiment, and also disappeared 30 minutes later. This indicates that, following destruction of the ventromedial hypothalamus, not only the inhibitory influence of this area upon activity of the 
B-cells disappears almost instantaneously but that the resulting hyper-reactivity of the endocrine pancreas remains unmodified subsequently. This study suggests that the rapid development of an overreactive endocrine pancreas may be of prime importance for the subsequent occurrence of obesity in animals with VMH lesions and that the hypothalamus appears to exert physiologically a minute to minute inhibitory influence upon insulin secretion. The neural or humoral nature of this hypothalamic regulation, as well as the precise anatomical localization of the hypothalamic area (s) responsible for it remain to be determined.

Acknowledgements. We are greatly indebted to Martha Bezemer for her excellent technical help. This work has been supported by grant 3.218.73 of the Fonds National Suisse de la Recherche Scientifique, Berne, Switzerland, and by a grant-in-aid of Nestlé Alimentana S. A., Vevey, Switzerland. This study has been carried out within the Fondation pour recherches médicales, Geneva, Switzerland.

\section{References}

1. Rabin, B.M.: Ventromedial hypothalamic control of food intake and satiety: A reappraisal. Brain Res. 43, 317-342 (1972)

2. Assimacopoulos-Jeannet, F., Jeanrenaud, B.: The hormonal and metabolic basis of experimental obesity. Clin. Endocrinol. Metabol. 5, 337-365 (1976)

3. Frohman, L.A., Bernardis, L. L., Schnatz, J.D., Burek, L.: Plasma insulin and triglyceride levels after hypothalamic lesions in weanling rats. Am. J. Physiol. 216, 1496-1501 (1969)

4. Martin, J. M., Konijnendîjk, W., Bowman, P. R.: Insulin and growth hormone secretion in rats with ventromedial hypothalamic lesions maintained on restricted food intake. Diabetes 23, 203-208 (1974)

5. Tannenbaum, G. A., Paxinos, G., Bindra, D.: Metabolic and endocrine aspects of the ventromedial hypothalamic syndrome in the rat. J. Comp. Physiol. Psychol. 86, 404-413 (1974)
6. Goldman, J.K., Bernardis, L.L., Frohman, L.A.: Food intake in hypothalamic obesity. Am. J. Physiol. 227, 88-91 (1974)

7. Karakash, C., Hustvedt, B.E., Løvø, A., Le Marchand, Y., Jeanrenaud, B.: Consequences of ventromedial hypothalamic lesions upon metabolism of perfused rat liver. Am. J. Physiol. (in press)

8. Hustvedt, B.E., Løvø, A.: Correlation between hyperinsulinemia and hyperphagia in rats with ventromedial hypothalamic lesions. Acta Physiol. Scand. 84, 29-33 (1972)

9. York, D. A., Bray, G. A.: Dependence of hypothalamic obesity on insulin, the pituitary and the adrenal gland. Endocrinology 90, 855-894 (1972)

10. Porte, D., Jr., Girardier, L., Seydoux, J., Kanazawa, Y., Posternak, J.: Neural regulation of insulin secretion in the dog. J. Clin. Invest. 52, 210-214 (1973)

11. Woods, S. C., Porte, D., Jr.: Neural control of the endocrine pancreas. Physiol. Rev. 54, 596-619 (1974)

12. Powley, T.L., Opsahl, C.A.: Ventromedial hypothalamic obesity abolished by subdiaphragmatic vagotomy. Am. J. Physiol. 226, 25-33 (1974)

13. Idahl, L. A., Martin, J. M.: Stimulation of insulin release by a ventrolateral hypothalamic factor. J. Endocrinol. 51, 601$602(1971)$

14. Martin, J. M., Mok, C. C., Penfold, J., Howard, N. J., Crowne, D.: Hypothalamic stimulation of insulin release. J. Endocrinol, 58, 681-682 (1973)

15. Curry, D.L., Joy, R.M.: Direct CNS modulation of insulin secretion. Endocr. Res. Commun. 1, 229-237 (1974)

16. De Groot, J.: The rat forebrain in stereotaxic coordinates. Trans. R. Neth. Acad. Sci. 52, 1-40 (1959)

17. Herbert, V., Lau, K.S., Gottlieb, C.W., Bleicher, S.J.: Coated charcoal immunoassay of insulin. J. Clin. Endocrinol. Metab. 25, 1375-1384 (1973)

18. Bergmeyer, H.U., Bernt, E.: In: H. U. Bergmeyer (Ed.) Methods of enzymatic analysis, pp. 123-130. New York: Academic Press 1963

19. Steffens, A.B., Mogenson, G.J., Stevenson, J.A. F.: Blood glucose, insulin, and free fatty acids after stimulation and lesions of the hypothalamus. Am. J. Physiol. 222, 1446-1452 (1972)

Received: November 25, 1976, and in revised form:

January 21, 1977

Dr. B. Jeanrenaud

Laboratoires de Recherches Médicales

64, avenue de la Roseraie

CH-1205 Geneva

Switzerland 